\title{
Spectral Synthesis of Cool Components of Symbiotic Stars
}

\author{
Claudio B. Pereira and Sayd Codina Landaberry \\ Observatório Nacional \\ Rua José Cristino, 77 São Cristóvão - CEP 20921 \\ Rio de Janeiro - Brasil
}

\begin{abstract}
We analyzed the optical spectra of cool component of symbiotic star SY Mus by means of spectral synthesis technique in order to derive the atomic abundances using the Minneart formulae for computing the atmospheric opacities. We obtained a satisfactory agreement between the observed and computed spectra and the resulting abundances were consistent to the solar abundances.
\end{abstract}

INTRODUCTION. The optical spectra of symbiotic stars contain various emission lines superimposed on the red continuum of the cool component. In these continuous spectra we observed molecular bands of TiO at $\lambda 5850 \AA, \lambda 6200 \AA$ and $\lambda 7200 \AA$ which characterize the spectra of the cool red giants. Sometimes is difficult to analyze the spectra of these cool components. This difficulty arises due the large strengths of Tio bands blanketing which strike out the atomic absorption lines or by the presence of the emission lines that blends it.

THE MODEL. We used as first approximation an isothermal model with a linear variation of the source function with the optical depth and the absorptions which are the only interactions between the photons and atoms and molecules. This model can be written by Minneart formulae as

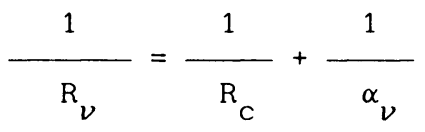

where

$R_{v}$, is the residual intensity;

$R_{C}$, is the central intensity and

$\alpha_{v}$, is the absorption coefficient per particle in the lower level of the transition.

OBSERVATIONS. Our study is based on spectra obtained at Laboratório Nacional de Astrofísica. The data were obtained with a coude spectrograph (first order) attached to the $1.6 \mathrm{~m}$ telescope. The detector used was a CCD with 576 pixels with a grating of $18 \AA / \mathrm{mm}$. We analyzed two regions of SY Mus; one between $5400 \AA$ and $5425 \AA$ and the other between $5430 \AA$ and $5445 \AA$. The raw data were corrected for flatfield and wavelength scale was established through the spectrum of a thorium lamp. 
RESULTS AND DISCUSSION. Despite of this simple model with one slab for the formation of absorption lines (atomic and molecular) the agreement between the computed and observed spectra was good. From our synthesis we found solar abundances in the region analyzed. Very little components of symbiotic stars had their spectra been analyzed in detail until now. The studies in this directions are still in the beginning. ER Del, (Johnson, H. R. and Ake, T.B., 1989) ; BD-21.3873, (Jorisson, A., 1989); R Aqr and UV Aur, (Little, Little-Marenin and Bauer, 1987) are good candidates to analyze if these stars have enhanced s-elements lines such us $\mathrm{Sr}, \mathrm{Y}, \mathrm{Ba}, \mathrm{Nd}$ and $\mathrm{Sm}$ in their spectra. Techenetium lines could also be investigated in cold giants of symbiotic stars. We plan to observe in the region between $4100 \AA$ and $4400 \AA$ where technetium lines of $4238 \AA, 4262 \AA$ and $4297 \AA$ are visible despite of low levelof spectral energy in the blue continuum. Some of them have period longer than 300 days (Whitelock, 1987) and mass of 3M॰ (Luud and Leédyarv, 1986) so we have to look for if some peculiar red giants could belongs to one of these symbiotic systems.

\section{References :}

Johnson, H. R. and Ake, T. (1989) 'BD-21.3873: An Heavy Element-Rich Symbiotic?', Evolution of Peculiar Red Giants Stars. IAU Colloquium, 106.

Jorissen, A. (1989) 'ER Del : A True Symbiotic S Star?', Evolution of Peculiar Red Giants Stars. IAU Colloquium, 106.

Little, S. J., Little-Marenin, I. R. and Bauer, W. (1987).

'Additional Late-Type Stars with Techenetium', Astron. J. 94,981-995

Luud, L and Leédyarv, L. (1986).'Properties of the Cold Components Of Symbiotic Stars', Astrophysics, 24, 154-162.

Whitelock, P. A. (1987), 'Symbiotic Miras', Publ. Ast. Soc. Pac. , 99, 573-591. 